\title{
Inestabilidad de la leche asociada a componentes lácteos y estacionalidad en vacas a pastoreo
}

\author{
Claudia Gabriella Barchiesi-Ferrari(1), Pamela Alejandra Williams-Salinas ${ }^{(2)}$ y Sonia Ilse Salvo-Garrido(3)
}

(1)Universidad de La Frontera (Ufro), Fac. de Ciencias Agropecuarias y Forestales, Dep. de Producción Agropecuaria, Av. Francisco Salazar 01145, Temuco, Chile. E-mail: cbarchi@ufro.cl (2)Universidad de Concepción, Fac. de Agronomía, Dep. de Producción Animal, Av. Vicente Méndez 595, Chillán, Chile. E-mail: pamelawilliams@udec.cl (3)Ufro, Fac. de Ingeniería, Ciencias y Administración, Dep. de Matemáticas y Estadística. E-mail: ssalvo@ufro.cl

\begin{abstract}
Resumen - El objetivo de este trabajo fue caracterizar rebaños lecheros que presentaban muestras de leche positivas a la prueba de alcohol sin estar ácidas, y compararlos con predios negativos a esta prueba, considerándose el efecto de la estacionalidad. Se estudiaron cuatro rebaños (tres positivos a la prueba de alcohol y uno negativo). En dos años, se muestreó leche durante tres meses en lactancias de invierno (época 1) y tres meses en lactancias de verano (época 2). En la dieta, se realizó análisis proximal relativo a EM, $\mathrm{Ca}, \mathrm{P}, \mathrm{Mg}$ y $\mathrm{K}$. En leche, se determinó proteína, lactosa, $\mathrm{Ca}, \mathrm{P}, \mathrm{Mg}, \mathrm{K}, \mathrm{pH}$, prueba de alcohol, acidez titulable y actividad proteásica. En casos negativos a la prueba de alcohol, se observó alta correlación entre proteína cruda láctea, proteína cruda en alimento, fósforo lácteo y lactosa. Los predios positivos a la prueba de alcohol se correlacionaron fuertemente con la menor concentración de materia seca y el alto contenido de fibra cruda, además presentaron menor concentración de proteína láctea. Ello indicaría que existió un efecto del manejo alimentario sobre la inestabilidad de la leche. Las lactancias desarrolladas durante la sequía estival sufren de un stress alimenticio, por lo cual podrían presentar inestabilidad láctea.
\end{abstract}

Términos para indexación: estabilidad de leche, composición láctea, producción estacional, pastoreo.

\section{Milk instability associated with milk composition and seasonal lactation in grazing dairy cows}

\begin{abstract}
The aim of this work was to characterize dairy herds which presented positive result for alcohol test in milk showing no acidity; and to compare them to a dairy herd with negative result for same test, considering the seasonal effect. Four dairy milk herds were studied (three positives to alcohol test and one with negative result). Milk samples were collected during 3 months in winter season (season 1) and 3 months in summer lactation (season 2) for 2 years. A subsample of the diets were prepared and submitted to proximal analysis for $\mathrm{ME}$ content, $\mathrm{Ca}, \mathrm{P}, \mathrm{Mg}$ and $\mathrm{K}$. Milk subsamples were analyzed for protein, lactose, $\mathrm{Ca}, \mathrm{Mg}, \mathrm{P}, \mathrm{pH}$, alcohol test, titratable acidity and proteasic activity. In cases negative to alcohol test, a high correlation index with milk crude protein, diet crude protein, milk phosphorus and lactose were observed. Dairy herds positive to alcohol test were highly correlated with lower dry matter content and a high content of crude fiber, besides these herds presented lower milk protein concentration. It was possible to notice an effect of feeding management in the instability of the milk. Summer's lactations under feeding stress condition would present milk instability.
\end{abstract}

Index terms: milk stability, milk composition, seasonal production, grazing.

\section{Introducción}

La estabilidad térmica se refiere a la capacidad de la leche para resistir a altas temperaturas de procesamiento, sin presentar coagulación o gelificación visibles (Singh, 2004). Entre los múltiples factores que ocasionan inestabilidad de la leche, se encuentran la raza (Hernández \& Ponce, 2005), el polimorfismo genético de la caseína (Williams, 2002; Raynal-Ljutovac et al., 2007), la composición de la leche (Williams, 2002; Hernández \& Ponce, 2005), el pH de la leche (Guo et al., 1998; O'Sullivan et al., 2001), el equilibrio mineral (Guo et al., 1998; Williams, 2002; Raynal-Ljutovac et al., 2007) y el tratamiento térmico (Williams, 2002; Hernández \& Ponce, 2005). 
La inestabilidad no sólo se debería a la actividad proteolítica, sino también a procesos fisicoquímicos en la micela de caseína (Venkatachalam et al., 1993). Es así como frecuentemente las muestras de leche resultan positivas a la prueba de alcohol, sin estar ácidas (Ponce \& Hernández, 2001). Esta prueba es aplicada en las plantas procesadoras, durante la recepción de la leche, para detectar problemas de termoestabilidad en la leche cruda (Guo et al., 1998; Raynal-Ljutovac et al., 2007). En ella, se puede observar la desestabilización coloidal de la micela de caseína, por el efecto desnaturalizador del alcohol (O'Connell et al., 2001).

A pesar de ser conocidos los factores de la industria y de la leche que ocasionan la inestabilidad, no hay claridad sobre cuales factores de manejo predial generarían dicha inestabilidad. Según Auldist et al. (2007), hay condiciones climáticas y ambientales que pueden afectar la estacionalidad de la producción de leche, no obstante existe escasa información sobre el impacto de estos factores en vacas a pastoreo. La calidad de la ración recibida por el rebaño lechero y, en particular, el tipo de proteína y el balance iónico cumplirían un rol relevante en la estabilidad de la leche.

El objetivo de este estudio fue caracterizar y relacionar los componentes nutricionales de la ración y la composición de la leche, en rebaños que normalmente producen leche positiva a la prueba de alcohol, en comparación con los resultados obtenidos para esas variables en un predio que produce leche negativa a dicha prueba. Como segundo objetivo se consideró analizar el efecto de la estación climática del año, en la presencia de inestabilidad de la leche.

\section{Material y Métodos}

El estudio se realizó en la zona de Loncoche, $39^{\circ} \mathrm{S}$, Chile, en rebaños Frisón Negro Chileno a pastoreo. La región posee suelos derivados de cenizas volcánicas modernas, con $2.000 \mathrm{~mm}$ de precipitación anual (1.600 mm entre otoño e invierno), temperatura media de suelo $11-12^{\circ} \mathrm{C}$.

Este estudio se realizó en cuatro lecherías, habiéndose comparado tres meses de las lactancias de invierno (época 1) con partos en otoño, y lactancias de verano (época 2) con partos en primavera, durante dos años, para tener igual período de evaluación en cada estación climática. Además, los registros de la planta procesadora de leche indicaron, para estos predios, un incremento de resultados positivos a la prueba de alcohol durante el período estival.
En la planta procesadora de leche se revisaron los registros diarios de la prueba de alcohol en leche, realizados en los estanques prediales. A partir de los registros se seleccionaron tres predios que producían leche positiva a la prueba de alcohol (PPA) y un predio con leche negativa a esta prueba (PNA), especialmente durante el período estival. En los predios seleccionados, se realizó la prueba de alcohol a cada una de las vacas del rebaño, para confirmar la inestabilidad de la leche. Luego, de los PPA se eligieron 10 vacas que dieron leche positiva al test, entre aquellas que presentaron más de ocho semanas de lactancia (media \pm EE) $(127 \pm 21$ y $140 \pm 17$ días, tanto en las lactancias de invierno y como de verano, respectivamente), lo que representó entre 10 y $24 \%$ del rebaño. En el PNA, se seleccionaron 10 vacas (17\% del rebaño) a igual período de lactancia (124 \pm 11 días), que resultaron negativas al test de alcohol.

Otros factores considerados en la selección de los predios fueron el porcentaje de "holsteinización" de los rebaños (entre 50 y 75\%), distribución de los partos (pariciones de otoño y primavera), recursos alimenticios empleados (pradera, ensilaje, heno, concentrados) y fechas de partos. Se recopiló información de manejo mediante encuestas, para apoyar la interpretación de los resultados.

Se tomaron muestras de alimentos, semanalmente, y se hicieron muestras compuestas mensuales en cada época y predio. Durante una semana en cada mes, el consumo en pastoreo se estimó mediante un plato medidor de forraje. Los otros componentes de la dieta (ensilaje, soiling, concentrados, minerales y aditivos) eran suministrados y mezclados en comederos. Entonces, la composición de la dieta fue reconstruida, a partir de los valores de estimación del consumo de materia seca en pastoreo y del consumo en comedero de los animales seleccionados en cada predio. Se analizaron las dietas compuestas de cada lechería para materia seca (MS), cenizas totales (CT), fibra cruda (FC), extracto no nitrogenado (ENN), fósforo por colorimetría (PA), calcio $(\mathrm{CaA})$, potasio $(\mathrm{KA})$ y magnesio $(\mathrm{MgA})$ por espectrofotometría de absorción atómica (Association of Official Analytical Chemists, 1996), proteína cruda por Kjeldahl (PCA), extracto etéreo (EE) según Association of Official Analytical Chemists (1996). El contenido de energía metabolizable (EM) fue calculado a partir de nutrientes digestibles totales. La composición nutricional por predio y época de año se presenta en el Cuadro 1.

Se tomaron muestras de leche individuales por semana, para luego hacer muestras compuestas proporcionales, por vaca, y una muestra por grupo por mes. 
Estas muestras compuestas de leche fueron analizadas: para $\mathrm{pH}$ por potenciometría $(\mathrm{pH})$; acidez titulable (ACD), por medio de $\mathrm{NaOH} 0,1 \mathrm{~N}$; prueba de alcohol (76\%); proteína cruda por Kjeldhal (PCL); contenido de lactosa (La), $\mathrm{Ca}(\mathrm{CaL}), \mathrm{Mg}(\mathrm{MgL})$ y $\mathrm{K}(\mathrm{KL})$, por espectrofotometría de absorción atómica; y $\mathrm{P}$ (PL), por colorimetría (Association of Official Analytical Chemists, 1996). Se determinó mensualmente la actividad proteásica en la leche, por liberación de p-nitroanilina a partir de un sustrato sintético (Costa et al., 2002).

Como análisis complementario en cada predio, se realizaron perfiles metabólicos de cada vaca en estudio, mensualmente, durante cada época evaluada. Se determinaron: la hemoglobina $(\mathrm{Hb})$, mediante el método de la cianometahemoglobina (Jain, 1986); la proteína, por refractometría; albúmina (Alb), por verde bromocresol; globulina (Glo), por diferencia entre proteína y $\mathrm{Alb}$; beta-hidroxibutirato (B-OHB), aspartatoamino-transferasa (Ast); urea; $\mathrm{P}$ inorgánico; $\mathrm{Ca}, \mathrm{Mg}$ (Tadich et al., 1997); K, por espectrofotometría de absorción atómica; glutation peroxidasa (GSH-Px), por método descrito por Ceballos et al. (1999).

Los datos obtenidos, en las distintas evaluaciones, fueron analizados mediante un análisis exploratorio con el fin de encontrar sus anomalías y presentar una caracterización de las variables; se realizó el análisis de correlaciones de Pearson, para ver el nivel de asociación entre ellas. Un análisis de varianza (ANDEVA) fue realizado con los componentes dietarios, para ver diferencias entre predios y época, a través de procedimiento GLM, con arreglo factorial con predio como factor principal y época como secundario. Se realizó un ANDEVA de una vía para componentes lácteos, para estudiar las diferencias entre predios.

\section{Resultados y Discusión}

En la región en estudio, los planteles lecheros basan su producción en pastoreo en praderas sin riego, por lo que la sequía estival afecta la disponibilidad y calidad de la pradera. Esto hace necesaria la suplementación, que muchas veces no permite alcanzar un balance adecuado de nutrientes.

En el Cuadro 1, se observa que el contenido de MS de las dietas ofrecidas no presentó diferencias $(p<0,05)$ entre predios, salvo en el predio 2 ( $26,0 \pm 3,7$ vs. $33,3 \pm 3,3$, época 1 y 2 , respectivamente), el cual, durante la época invernal, no dispuso de suficiente ensilaje de pradera para paliar la escasez de forraje para pastoreo. La falta de este ingrediente pudo influir en la diferencia de la composición nutricional de este predio entre verano e invierno. En relación a la FC, en los predios 1, 2 y 3 aumentó su porcentaje dietario en la época 2 (6, 12 y $33 \%$, respectivamente), lo cual podría reflejar un deterioro de la calidad de la pradera debido a la sequía estival. Sin embargo, esto no sucedió en el predio 4. En el mismo Cuadro 1, se puede observar que el contenido de $\mathrm{Ca}$, ofrecido en los distintos predios, no satisface la concentración mínima de Ca recomendada. Esto concuerda con lo obtenido por Cuevas et al. (1983), quienes, plantean que el contenido de leguminosas de las praderas del Sur de Chile es bajo y, por ende, el aporte de Ca. Por este motivo, en los predios 2, 3 y 4 se utilizó suplementación con sales minerales en distinta proporción.

La información presentada en el Cuadro 2 corresponde a la composición láctea de los predios (media土EE); no se consideró la diferenciación por época por no ser estadísticamente significativa en las variables

Cuadro 1. Composición de la dieta suministrada a los predios en cada época (lactancias de invierno y verano), expresados como media y error estándar ${ }^{(1)}$.

\begin{tabular}{|c|c|c|c|c|c|c|c|c|c|c|c|}
\hline \multirow[t]{2}{*}{ Composición } & \multicolumn{2}{|c|}{ Predio 1} & \multicolumn{2}{|c|}{ Predio 2} & \multicolumn{2}{|c|}{ Predio 3} & \multicolumn{2}{|c|}{ Predio 4} & \multicolumn{3}{|c|}{ Probabilidad } \\
\hline & Época 1 & Época 2 & Época 1 & Época 2 & Época 1 & Época 2 & Época 1 & Época 2 & $\mathrm{P}$ & $\mathrm{E}$ & PxE \\
\hline$\overline{M S}(\%)$ & $26,8 \pm 2,0$ & $24,6 \pm 1,6$ & $26,0 \pm 3,7$ & $33,3 \pm 3,3$ & $34,9 \pm 3,6$ & $32,9 \pm 3,6$ & $33,2 \pm 2,5$ & $30,6 \pm 2,0$ & * & & \\
\hline CT $(\%)$ & $6,77 \pm 0,2$ & $7,2 \pm 0,5$ & $7,63 \pm 0,4$ & $6,74 \pm 0,5$ & $8,17 \pm 0,7$ & $7,17 \pm 0,2$ & $5,97 \pm 0,3$ & $6,4 \pm 0,6$ & * & & \\
\hline $\mathrm{PC}(\%)$ & $11,0 \pm 0,5$ & $11,1 \pm 0,6$ & $16,3 \pm 0,7$ & $8,3 \pm 0,3$ & $13,4 \pm 0,3$ & $10,6 \pm 1,6$ & $13,6 \pm 0,8$ & $14,6 \pm 1,5$ & * & $*$ & $*$ \\
\hline $\mathrm{FC}(\%)$ & $26,6 \pm 1,7$ & $28,3 \pm 0,9$ & $22,7 \pm 1,8$ & $25,8 \pm 1,3$ & $18,0 \pm 1,4$ & $26,8 \pm 1,5$ & $21,8 \pm 1,6$ & $19,8 \pm 1,8$ & $*$ & $*$ & \\
\hline $\mathrm{EE}(\%)$ & $2,63 \pm 0,4$ & $4,37 \pm 1,3$ & $3,43 \pm 0,5$ & $3,30 \pm 1,2$ & $2,43 \pm 0,4$ & $3,13 \pm 1,2$ & $5,40 \pm 1,0$ & $4,53 \pm 1,2$ & & & \\
\hline ENN (\%) & $51,7 \pm 2,7$ & $47,3 \pm 2,9$ & $56,1 \pm 7,0$ & $55,8 \pm 1,3$ & $55,6 \pm 0,1$ & $53,4 \pm 2,9$ & $50,8 \pm 3,1$ & $52,8 \pm 2,0$ & & & \\
\hline $\mathrm{EM}\left(\right.$ Mcal kg$\left.{ }^{-1}\right)$ & $2,5 \pm 0,0$ & $2,5 \pm 0,0$ & $2,69 \pm 0,2$ & $2,52 \pm 0,0$ & $2,50 \pm 0,0$ & $2,48 \pm 0,0$ & $2,61 \pm 0,0$ & $2,55 \pm 0,0$ & & & \\
\hline $\mathrm{Ca}(\mathrm{mg} / 100 \mathrm{~g})$ & $576 \pm 39$ & $501 \pm 71$ & $528 \pm 70$ & $570 \pm 76$ & $1.453 \pm 386$ & $562 \pm 91$ & $1.093 \pm 597$ & $650 \pm 177$ & $*$ & & \\
\hline $\mathrm{P}(\mathrm{mg} / 100 \mathrm{~g})$ & $348 \pm 30$ & $308 \pm 41$ & $408 \pm 11$ & $245 \pm 41$ & $710 \pm 198$ & $236 \pm 29$ & $398 \pm 28$ & $427 \pm 40$ & & & \\
\hline $\mathrm{Mg}(\mathrm{mg} / 100 \mathrm{~g})$ & $203 \pm 7$ & $188 \pm 21$ & $237 \pm 20$ & $217 \pm 14$ & $499 \pm 185$ & $223 \pm 17$ & $224 \pm 12$ & $256 \pm 30$ & & & \\
\hline $\mathrm{K}(\mathrm{mg} / 100 \mathrm{~g})$ & $1.408 \pm 30$ & $1.680 \pm 269$ & $1.442 \pm 160$ & $705 \pm 14$ & $1.460 \pm 162$ & $1.108 \pm 217$ & $1.188 \pm 125$ & $1.638 \pm 223$ & * & * & \\
\hline
\end{tabular}

(1) Época 1: lactancia de invierno de vacas paridas en otoño; época 2: lactancia de verano de vacas paridas en primavera; P = predio 1, 2, 3 y 4; predios 1, 2 y 3 son positivos a la prueba de alcohol, y predio 4 es negativo a dicha prueba; $\mathrm{E}=$ época; PxE = predio $\mathrm{x}$ época; los valores de CT, EE y ENN fueron obtenidos de $\mathrm{n}=3$, mientras que los otros componentes de la composición dietaria corresponden al promedio de $\mathrm{n}=6$. $*$ Significativo a $5 \%$ de probabilidad. 
de interés. La menor concentración de PCL la presentó el predio $3(2,74 \%, \mathrm{p}<0,05)$, valor similar a $2,8 \%$, obtenido por Hernández \& Ponce (2005) al establecer una restricción alimenticia de $20 \%$ del consumo de MS, en vacas Holstein en Cuba; y menor al valor de 3,02\% reportado por Zanela et al. (2006a), para vacas Holstein en Brasil. Ponce \& Hernández (2001) caracterizaron el "síndrome de leche anormal" (SILA) bajo las condiciones de manejo de Cuba. Los autores mencionados señalaron como característica la alteración de los sólidos y reportaron valores de proteína láctea menor o igual a $2,9 \%$, lo cual concuerda con lo encontrado en este trabajo para los predios 1 y 3 . En relación a La, todos los predios se encontraron sobre el nivel de referencia de $4,6 \%$ de La para clasificar un predio SILA. La mayor concentración de PL la alcanzó el predio 4 (84 mg por $100 \mathrm{~g}, \mathrm{p}<0,05)$, superando ligeramente el referencial de $80 \mathrm{mg}$ por $100 \mathrm{~g}$ del SILA.

Según Hernández \& Ponce (2005), cuando existe una alta concentración láctea de Ca y el pH es inferior a 6,7, el Ca y citrato forman un complejo soluble estable, lo cual hace a la leche más estable. En cambio, si el pH de la leche es elevado y disminuye la concentración de Ca, se presenta una disminución de la concentración de citrato en su forma soluble (Hernández \& Ponce, 2005). En el Cuadro 2, a pesar de no observarse valores de $\mathrm{pH}$ lácteos alterados, se observó una baja concentración de Ca respecto a los valores mencionados en el tratamiento control, sin restricción alimentaria, que plantearon los autores previamente mencionados (114 g por $100 \mathrm{~g}$ ), lo cual podría afectar la formación de citrato, por ende, la estabilidad de la leche.

Durante mucho tiempo, la inestabilidad de la leche ha sido explicada por la actividad proteásica o enzimática (Venkatachalam et al., 1993). El predio 2, que fue seleccionado por producir leche inestable, presentó menor actividad enzimática que los otros 3 predios (Cuadro 2) y resultó ser positivo a la prueba de alcohol. En el Cuadro 2, se puede observar que el predio 4 no difirió estadísticamente de la actividad enzimática que presentaron los predios 1 y 3 ( $\mathrm{p}<0,05)$, los cuales son PPA. Por lo que no parece adecuado que la actividad enzimática, por si sola, indique inestabilidad de la leche.

En el Cuadro 3, se presentan los perfiles metabólicos (media $\pm E E$ ) de las vacas en estudio, por predio y época

Cuadro 2. Valores promedio y error estándar (media $\pm E E$ ) de componentes lácteos, predios positivos y negativos a la prueba de alcohol $(\%)^{(1)}$.

\begin{tabular}{|c|c|c|c|c|c|}
\hline Componente & Predio 1 & Predio 2 & Predio 3 & Predio 4 & $\mathrm{p}$ \\
\hline Proteína (\%) & $2,88 \pm 0,04 \mathrm{ab}$ & $3,06 \pm 0,12 \mathrm{a}$ & $2,74 \pm 0,06 b$ & $2,99 \pm 0,05 a$ & * \\
\hline Calcio (mg por $100 \mathrm{~g}$ ) & $97,78 \pm 3,10$ & $111,33 \pm 2,42$ & $98,43 \pm 4,44$ & $100,50 \pm 3,01$ & \\
\hline Fósforo $(\mathrm{mg}$ por $100 \mathrm{~g})$ & $76,80 \pm 2,03 \mathrm{ab}$ & $78,69 \pm 4,13 \mathrm{ab}$ & $71,93 \pm 3,15 b$ & $84,62 \pm 2,27 \mathrm{a}$ & * \\
\hline Magnesio (mg por $100 \mathrm{~g}$ ) & $9,87 \pm 0,43$ & $10,01 \pm 0,46$ & $8,92 \pm 0,28$ & $9,84 \pm 0,19$ & \\
\hline Potasio $(\mathrm{mg}$ por $100 \mathrm{~g})$ & $125,0 \pm 2,71$ & $129,5 \pm 6,01$ & $117,0 \pm 4,01$ & $120,2 \pm 4,16$ & \\
\hline Lactosa $(\%)$ & $4,83 \pm 0,09$ & $4,84 \pm 0,18$ & $4,69 \pm 0,21$ & $5,03 \pm 0,11$ & \\
\hline $\mathrm{pH}$ & $6,57 \pm 0,07$ & $6,69 \pm 0,10$ & $6,58 \pm 0,10$ & $6,61 \pm 0,04$ & \\
\hline Acidez ( ${ }^{\circ}$ Dornic) & $19,67 \pm 0,37 \mathrm{ab}$ & $20,43 \pm 0,52 a$ & $17,70 \pm 0,66 \mathrm{~b}$ & $20,45 \pm 0,47 \mathrm{a}$ & * \\
\hline Positivos a prueba de alcohol (\%) & 74,6 & 33,3 & 35,2 & 10,1 & \\
\hline Actividad proteásica ( $\mu \mathrm{m}$ de $\mathrm{p} \mathrm{Na} / 2 \mathrm{~h}$ ) & $44,44 \pm 3,27 \mathrm{a}$ & $25,7 \pm 2,93 b$ & $34,71 \pm 2,95 \mathrm{ab}$ & $34,68 \pm 3,77 \mathrm{ab}$ & * \\
\hline
\end{tabular}

${ }^{(1)}$ Medias en una misma línea, seguidas de letra diferente, indican diferencias significativas en test de Tukey, con $5 \%$ de probabilidad.

Cuadro 3. Valores promedio y error estándar (media $\pm E E)$ de perfiles metabólicos de los predios, según época de lactancia ${ }^{(1)}$.

\begin{tabular}{|c|c|c|c|c|c|c|c|c|}
\hline \multirow[t]{2}{*}{ Perfil metabólico } & \multicolumn{2}{|c|}{ Predio 1} & \multicolumn{2}{|c|}{ Predio 2} & \multicolumn{2}{|c|}{ Predio 3} & \multicolumn{2}{|c|}{ Predio 4} \\
\hline & Época 1 & Época 2 & Época 1 & Época 2 & Época 1 & Época 2 & Época 1 & Época 2 \\
\hline $\mathrm{Hb}^{(2)}\left(\mathrm{g} \mathrm{dl}^{-1}\right)$ & $9,75 \pm 0,21$ & $10,94 \pm 0,24$ & $10,11 \pm 0,22$ & $11,04 \pm 0,26$ & $11,01 \pm 0,2$ & $10,84 \pm 0,27$ & $9,93 \pm 0,33$ & $10,74 \pm 0,27$ \\
\hline Urea $\left(\mathrm{mmol} \mathrm{L}^{-1}\right)$ & $2,33 \pm 0,14$ & $4,06 \pm 0,29$ & $6,08 \pm 0,24$ & $2,66 \pm 0,23$ & $4,01 \pm 0,23$ & $2,65 \pm 1,67$ & $2,15 \pm 0,21$ & $3,57 \pm 0,10$ \\
\hline Proteína $\left(\mathrm{g} \mathrm{L}^{-1}\right)$ & $75,8 \pm 4,69$ & $75,0 \pm 1,20$ & $72 \pm 2,44$ & $77,5 \pm 1,87$ & $75,38 \pm 1,28$ & $72 \pm 1,33$ & $74 \pm 0,92$ & $77,80 \pm 1,27$ \\
\hline Albúmina $\left(\mathrm{g} \mathrm{L}^{-1}\right)$ & $40 \pm 1,47$ & $38,5 \pm 1,00$ & $34,1 \pm 0,75$ & $38,03 \pm 0,97$ & $38,38 \pm 1,03$ & $33,57 \pm 0,86$ & $39,17 \pm 2,86$ & $34,40 \pm 0,86$ \\
\hline Globulina $\left(\mathrm{g} \mathrm{L}^{-1}\right)$ & $35,7 \pm 5,32$ & $37 \pm 1,52$ & $38,00 \pm 2,88$ & $39,47 \pm 2,52$ & $37 \pm 1,09$ & $38,67 \pm 1,38$ & $34,83 \pm 2,74$ & $43,4 \pm 1,54$ \\
\hline $\mathrm{B}-\mathrm{OHB}{ }^{(3)}\left(\mathrm{mmol} \mathrm{L}^{-1}\right)$ & $0,54 \pm 0,05$ & $0,69 \pm 0,05$ & $0,68 \pm 0,04$ & $0,38 \pm 0,03$ & $1,01 \pm 0,15$ & $0,59 \pm 0,04$ & $0,71 \pm 0,05$ & $0,56 \pm 0,02$ \\
\hline GSH-Px ${ }^{(4)}\left(\mathrm{u} \mathrm{g}^{-1} \mathrm{Hb}\right)$ & $184 \pm 10,46$ & $185 \pm 18,43$ & $55,2 \pm 5,67$ & $113,7 \pm 11,53$ & $14,01 \pm 1,94$ & $39,5 \pm 4,60$ & $263,1 \pm 19,79$ & $262,5 \pm 5,31$ \\
\hline $\operatorname{Ast}^{(5)}\left(\mathrm{UL} \mathrm{L}^{-1}\right)$ & $99 \pm 4,54$ & $123 \pm 8,27$ & $101 \pm 2,74$ & $149,8 \pm 4,97$ & $105,9 \pm 4,79$ & $119,6 \pm 5,64$ & $103,9 \pm 5,12$ & $142,3 \pm 4,68$ \\
\hline $\mathrm{Ca}\left(\mathrm{mmol} \mathrm{L}^{-1}\right)$ & $2,2 \pm 0,04$ & $2,28 \pm 0,05$ & $2,23 \pm 0,06$ & $2,38 \pm 0,04$ & $2,46 \pm 0,04$ & $2,21 \pm 0,04$ & $2,44 \pm 0,06$ & $2,27 \pm 0,03$ \\
\hline $\mathrm{P}\left(\mathrm{mmol} \mathrm{L}^{-1}\right)$ & $1,76 \pm 0,10$ & $2,24 \pm 0,11$ & $1,95 \pm 0,07$ & $1,88 \pm 0,09$ & $1,34 \pm 0,07$ & $2,56 \pm 0,07$ & $1,90 \pm 0,13$ & $2,32 \pm 0,09$ \\
\hline $\operatorname{Mg}\left(\mathrm{mmol} \mathrm{L}^{-1}\right)$ & $0,94 \pm 0,03$ & $1,25 \pm 0,05$ & $0,73 \pm 0,03$ & $1,2 \pm 0,07$ & $0,88 \pm 0,02$ & $1,17 \pm 0,03$ & $0,84 \pm 0,08$ & $1,29 \pm 0,04$ \\
\hline $\mathrm{K}\left(\mathrm{mmol} \mathrm{L}^{-1}\right)$ & $5,44 \pm 0,21$ & $7,0 \pm 0,29$ & $4,60 \pm 0,13$ & $7,47 \pm 0,32$ & $6,06 \pm 0,20$ & $6,43 \pm 0,23$ & $5,86 \pm 0,19$ & $6,91 \pm 0,43$ \\
\hline
\end{tabular}

(1) Promedio de 10 muestreos en cada época; época 1: lactancia de invierno de vacas paridas en otoño; época 2: lactancia de verano de vacas paridas en primavera. ${ }^{(2)}$ Hemoglobina. ${ }^{(3)}$ Beta-hidroxibutirato. ${ }^{(4)}$ Glutation peroxidasa. ${ }^{(5)}$ Aspartato aminotransferasa. 
del año. Durante el estudio los rebaños, se encontraban en homeostasis, lo cual es ratificado por los valores de $\mathrm{Hb}$, urea, Alb y Glo. El predio 3, en su lactancia invernal, mostró una tendencia a mayor movilización de grasa, al presentar un contenido de B-OHB mayor, respecto de los otros predios en igual época $\left(1,01 \pm 0,15 \mathrm{mmol} \mathrm{L}^{-1}\right)$, esto pudo deberse a que el mayor contenido de cenizas dietarios afecta negativamente la digestibilidad de los nutrientes, y por ende su disponibilidad de energía. El P tuvo una menor concentración en el período invernal, respecto a época 2, salvo en el predio 2 . Respecto a ello, Hernández \& Ponce (2005) señalan que si la disponibilidad fisiológica de $\mathrm{P}$ es baja, también lo será en la glándula mamaria, lo que afecta la disponibilidad de $\mathrm{Ca}$, de P solubles y de fosfato cálcico micelar, que inciden en la inestabilidad de la leche.

En el Cuadro 4, se puede observar el coeficiente de correlación que existe entre los componentes dietarios y lácteos. Los componentes PCL y La, PCL con PL, PCA con La presentaron mayor correlación entre sí. Ello podría explicarse por una mayor disponibilidad de energía para los tejidos epiteliales mamarios, lo que según Ponce \& Hernández (2001) implicaría la no ocurrencia de SILA. Los PNA presentaron mayor concentración de MS y mayor concentración de PL. Además, se encontró una alta correlación con menores concentraciones de FC en la dieta $(r=-0,56, p<0,01)$.

En PPA se presentó una alta correlación con una menor concentración de MS y con el contenido de FC de la dieta $(r=0,57, p<0,01)$. Esta relación podría explicarse, porque una elevada concentración de FC en la dieta conduciría a un alto nivel de acetato y alto contenido de proteína soluble en el rumen, acompañada de una baja concentración de PCA, lo que llevaría a una menor síntesis de proteína microbiana. Walker et al. (2004) plantean que vacas que consumen concentrados amiláceos pueden aumentar la ingesta de EM y de la concentración de proteína láctea, sin embargo la proteína metabolizable puede ser limitante en esos sistemas. Además, White (2001) plantea que cuando la calidad nutritiva de los forrajes es baja, disminuye la concentración de proteína láctea. Asimismo, un menor consumo o cambios en la calidad de la pradera pueden reducir la disponibilidad de aminoácidos para la síntesis de caseína (Auldist et al., 1998). En este estudio, no se analizó la caseína, sin embargo se encontró un efecto detrimental de la concentración de proteína total de la leche en los PPA, los cuales además se relacionan con baja concentración de PCA.

Los elementos Ca y P presentan un rol complejo en la estabilidad térmica de la leche, debido a que se distribuyen entre las fases coloidal y del suero de la leche (Williams, 2002). Existe una redistribución de las sales, de una fase a outra, que puede producir una redistribución de la proteína caseínica (Williams, 2002), por lo que una disminución en la disponibilidad de la pradera puede aumentar la proporción de proteínas del suero.

Ponce \& Hernández (2001) plantean que en aquellas zonas tropicales donde se localizan las razas lecheras más especializadas, las alteraciones en la composición

Cuadro 4. Correlaciones de Pearson entre variables lácteas y dietarias más importantes ${ }^{(1)}$.

\begin{tabular}{|c|c|c|c|c|c|c|c|c|c|c|c|c|}
\hline & $\mathrm{La}$ & PCL & PPA & PNA & $\mathrm{ACD}$ & MSA & PCA & $\mathrm{FC}$ & $\mathrm{CaA}$ & $\mathrm{MgA}$ & KA & PA \\
\hline$\overline{\mathrm{CaL}}$ & & & & & & & & & $0,40 * *$ & & & \\
\hline PL & $0,54 * *$ & $0,50 * *$ & $-0,49 * *$ & $0,48 * *$ & $0,51 * *$ & & & $-0,36^{*}$ & & & & \\
\hline $\mathrm{MgL}$ & & & & & $0,37^{*}$ & & & & & & & \\
\hline $\mathrm{KL}$ & $0,44 * *$ & & & & & & $0,37^{*}$ & & & & & \\
\hline $\mathrm{La}$ & 1 & $0,32 *$ & & & $0,38 *$ & & $0,42 * *$ & $-0,44 * *$ & & & $0,32 *$ & \\
\hline PCL & & 1 & & & $0,50 * *$ & & & $-0,31^{*}$ & & & & \\
\hline PPA & & & 1 & $0,99 * *$ & & $-0,36^{*}$ & & $0,57 * *$ & & & & \\
\hline PNA & & & & 1 & & $0,38^{*}$ & & $-0,56 * *$ & & & & \\
\hline $\mathrm{ACD}$ & & & & & 1 & & & & & & & \\
\hline MSA & & & & & & 1 & & $-0,35^{*}$ & $0,36^{*}$ & & $-0,48 * *$ & \\
\hline PCA & & & & & & & 1 & $-0,53 * *$ & & & $0,51 * *$ & \\
\hline FC & & & & & & & & 1 & $-0,53 * *$ & $-0,43 * *$ & & \\
\hline $\mathrm{CaA}$ & & & & & & & & & 1 & $0,61 * *$ & & $0,68 * *$ \\
\hline $\mathrm{MgA}$ & & & & & & & & & & 1 & & $0,89 * *$ \\
\hline KA & & & & & & & & & & & 1 & \\
\hline PA & & & & & & & & & & & & 1 \\
\hline
\end{tabular}

${ }^{(1)} \mathrm{CaL}$ : calcio lácteo; PL: fósforo lácteo; MgL: magnesio lácteo; KL: potasio lácteo; La: lactosa; PCL: proteína láctea; PPA: predios positivos a la prueba de alcohol; PNA: predios negativos a la prueba de alcohol; ACD: acidez; MS: materia seca alimento; PCA: proteína cruda alimento; FC: fibra cruda; CaA: calcio alimento; MgA: magnesio alimento; KA: potasio alimento; PA: fósforo alimento; blancos indican correlaciones no significativas. $*$ y **Significativa al nivel de 5 y $1 \%$ de probabilidad, respectivamente. 
láctea se observan con frecuencia durante la época de sequía. Lo anterior es concordante con lo observado en la zona Sur de Chile, donde se ha introducido la raza Holstein Friesian.

A partir de los resultados de esta caracterización, la inestabilidad de leche en Chile se presentaría con valores de algunos parámetros diferentes a los descritos por Ponce \& Hernández (2001), para el SILA en Cuba, y Zanela et al. (2006b) en Brasil. Por ello, es necesario seguir investigando para definir valores de referencia de los parámetros que inciden en la inestabilidad de leche, de acuerdo a las condiciones de suelo, clima, manejo alimenticio y genético animal en la zona Sur de Chile.

\section{Conclusiones}

1. Existe una estrecha relación entre los componentes nutricionales de la ración, sobre todo fibra cruda y materia seca, e incidencia de prueba de alcohol positiva.

2. Se observó en los casos negativos a la prueba de alcohol una alta correlación entre el contenido de materia seca y mayor concentración de fósforo lácteo, además de una alta correlación con menores concentraciones de fibra cruda en la dieta.

3. Los componentes proteína cruda láctea y lactosa, proteína cruda láctea con fósforo lácteo, proteína cruda del alimento con lactosa presentan mayores correlaciones entre sí.

4. Las lactancias que se desarrollan durante el período de sequía estival sufren un mayor stress alimenticio, por lo cual son más susceptibles a presentar inestabilidad láctea.

\section{Agradecimientos}

A la Universidad de La Frontera, por el financiamento del proyecto; al Prof. Horacio Miranda, por las sugerencias durante el desarrollo de esta investigación.

\section{Referencias}

ASSOCIATION OF OFFICIAL ANALYTICAL CHEMISTS. Official methods of analysis. $16^{\text {th }}$ ed. Washington: AOAC, 1996.

AULDIST, M.J.; TURNER, S.A.; MCMAHON, C.D.; PROSSER, C.G. Effects of melatonin on the yield and composition of milk from grazing dairy cows in New Zealand. Journal of Dairy Research, v.74, p.52-57, 2007.

AULDIST, M.J.; WALSH, B.J.; THOMSON, N.A. Seasonal and lactational influences on bovine milk composition in
New Zealand. Journal of Dairy Research, v.65, p.401411, 1998.

CEBALLOS, A.; WITTWER, F.G.; CONTRERAS, P.A.; QUIROZ, A.; BÖHMWALD, H.L. Actividad de glutation peroxidasa en bovinos lecheros a pastoreo correlacionada con la concentración sanguínea y plasmática de selenio. Pesquisa Agropecuária Brasileira, v.34, p.2331-2338, 1999.

COSTA-LOBO, M.; GOMEZ, M.F.; MOLINA, L.H.; SIMPSON, R.; ROMERO, A. Purificación y caracterización de proteasas de Pseudomonas fluorescens y sus efectos sobre las proteínas de la leche. Archivos Latinoamericanos de Nutrición, v.52, p.160-166, 2002.

CUEVAS, E.; BALOCCHI, O.; ANRIQUE, R.; JORQUERA, M. Valor nutritivo de las principales especies de una pradera permanente en la décima región: II. contenido de minerales. AgroSur, v.11, p.13$22,1983$.

GUO, M.; WANG, S.; LI, Z.; QU, J.; JIN, L.; KINDSTEDT, P. Ethanol stability of goat's milk: review 1968-1979. International Dairy Journal, v.8, p.57-60, 1998.

HERNÁNDEZ, R.; PONCE, P. Efecto de tres tipos de dieta sobre la aparición de trastornos metabólicos y su relación con alteraciones en la composición de la leche en vacas Holstein Friesian. Zootecnia Tropical, v.23, p.295-310, 2005.

JAIN, N.C. Schalm's Veterinary hematology. $4^{\text {th }}$ ed. Philadelphia: Lea \& Febiger, 1986. 1221p.

O'CONNELL, J.E.; KELLY, A.L.; FOX, P.F.; KRUIF, K.G. Mechanism for the ethanol-dependent heat-induced dissociation of casein micelles. Journal of Agricultural and Food Chemistry, v.49, p.4424-4428, 2001.

O'SULLIVAN, M.M.; LORENZEN, P.C.; O'CONNELL, J.E.; KELLY, A.L.; SCHLIMME, E.; FOX, P.F. Short communication: influence of transglutaminase on the heat stability of milk. Journal of Dairy Science, v.84, p.1331-1334, 2001.

PONCE, P.; HERNÁNDEZ, R. Propriedades físico-químicas do leite e sua associação com transtornos metabólicos e alterações na glândula mamária. In: GONZÁLEZ, F.H.D.; DÜRR, J.W.; FONTANELI, R.S. (Ed.). Uso do leite para monitorar a nutrição e metabolismo de vacas leiteiras. Porto Alegre: UFRGS, 2001. p.61-72.

RAYNAL-LJUTOVAC, K.; PARK, Y.W.; GAUCHERON, F.; BOUHALLAB, S. Heat stability and enzymatic modifications of goat and sheep milk. Small Ruminant Research, v.68, p.207-220, 2007.

SINGH, H. Heat stability of milk. International Journal of Dairy Technology, v.57, p.111-119, 2004.

TADICH, N.; MENDEZ, G.; WITTWER, F.; MEYER, K. Valores bioquímicos sanguíneos de equinos que tiran carretones en la ciudad de Valdivia (Chile). Archivos de Medicina Veterinaria, v.29, p.4561, 1997.

VENKATACHALAM, N.; MCMAHON, D.; SAVELLO, P.A. Role of protein and lactose interactions in the age gelation of ultra-high temperature processed concentrated skim milk. Journal of Dairy Science, v.76, p.1882-1894, 1993. 
WALKER, G.P.; DUNSHEA, F.R.; DOYLE, P.A. Effects of nutrition and management on the production and composition of milk fat and protein: a review. Australian Journal of Agricultural Research, v.55, p.1009-1028, 2004.

WHITE, C.L. Factors affecting milk protein concentration in Australian dairy cows. The Australian Journal of Dairy Technology, v.56, p.153, 2001.

WILLIAMS, R.P.W. The relationship between the composition of milk and the properties of bulk milk products. The Australian Journal of Dairy Technology, v.57, p.30-44, 2002.
ZANELA, M.B.; FISCHER, V.; RIBEIRO, M.E.R.; BARBOSA, R.; MARQUES, L.V.; STUMPF JÚNIOR,W.; ZANELA, C. Unstable nonacid milk and milk composition of Jersey cows on feed restriction. Pesquisa Agropecuária Brasileira, v.41, p.835-840, 2006b.

ZANELA, M.B.; FISCHER, V.; RIBEIRO, M.E.R.; STUMPF JÚNIOR, W.; ZANELA, C.; MARQUES, L.T.; MARTINS, P.R.G. Milk quality in production systems in the Southern region of Rio Grande do Sul, Brazil. Pesquisa Agropecuária Brasileira, v.41, p.153-159, 2006a.

Recibido el 23 de agosto de 2007 y aceptado el 21 de noviembre de 2007 